\title{
SCCA, TSGF, and the Long Non-Coding RNA AC007271.3 are Effective Biomarkers for Diagnosing Oral Squamous Cell Carcinoma
}

\author{
Tingru Shao ${ }^{\mathrm{a}}$ Jiaxin Huang ${ }^{\mathrm{a}}$ Zenan Zheng ${ }^{\mathrm{a}}$ Qingqing Wu $\mathrm{W}^{\mathrm{a}}$ Tiancai Liu ${ }^{\mathrm{b}}$ \\ Xiaozhi Lva \\ aDepartment of Oral \& Maxillofacial Surgery, NanFang Hospital, Southern Medical University, \\ Guangzhou, 'State Key Laboratory of Organ Failure, Institute of Antibody Engineering, School of \\ Laboratory Medicine and Biotechnology, Southern Medical University, Guangzhou, China
}

\section{Key Words}

Oral squamous cell carcinoma • Long non-coding RNA AC007271.3 • Microarray • Biomarker

- Squamous cell carcinoma antigen

\begin{abstract}
Background/Aims: Oral squamous cell carcinoma (OSCC) is one of the most lethal malignancies worldwide and the most common type of oral cancer, characterized by invasive growth, frequent regional metastases, high recurrence, and poor prognosis. In the current study, we investigated the use of long non-coding RNAs (IncRNAs), tumor-specific growth factor (TSGF), and squamous cell carcinoma antigen (SCCA) as potential biomarkers for OSCC screening. Methods: LncRNA expression was measured by microarray analysis in three sets of OSCC and paired normal mucosal tissues. The potential IncRNAs involved in OSCC development were investigated by bioinformatics and verification experiments. We also determined the expression of these potential biomarkers in tissue and serum samples in a case-control study of 80 OSCC cases and 70 controls. Receiver operating characteristics, decision curve analysis, and the combined detection of IncRNA AC007271.3, TSGF, and SCCA were carried out to screen for OSCC biomarkers. Results: A total of 691 IncRNAs (433 upregulated and 258 downregulated) were differentially expressed in OSCC tissues compared with normal controls $(p<0.05)$. Based on Gene Ontology and pathway analysis, we selected four differentially expressed IncRNAs (AC007271.3, AC007182.6, LOC283481, and RP11-893F2.9), and showed that aberrant AC007271.3 levels in OSCC patients were significantly associated with clinical stage, especially in early-stage disease, in an expanded case-control study. The combination of AC007271.3 and SCCA (AUC $=0.902, p<0.001$ ) showed significantly better ability to discriminate between OSCC and controls compared with SCCA or AC007271.3 alone. Serum AC007271.3, SCCA, and TSGF levels could also discriminate between OSCC and normal controls with sensitivities of $77.6 \%$, $55.0 \%$, and $63.3 \%$, and specificities of $84.5 \%, 93.3 \%$, and $66.7 \%$, respectively. Conclusions: These results suggest that AC007271.3, SCCA, and TSGF could be novel circulating biomarkers for the determination of OSCC. However, further validation in large-scale prospective studies is necessary.
\end{abstract}




\section{Cellular Physiology Cell Physiol Biochem 2018;47:26-38 and Biochemistry Published \begin{tabular}{l|l} 
DOI: 10.1159/000489741 & $\begin{array}{l}\text { C } 2018 \text { The Author(s). Published by S. Karger AG, Basel } \\
\text { www.karger.com/cpb }\end{array}$
\end{tabular} Shao et al.: Circulating Biomarkers in OSCC}

\section{Introduction}

Oral squamous cell carcinoma (OSCC) is one of the most lethal malignancies and is also the most common oral cancer, accounting for $95 \%$ of all oral cancer diagnoses and causing more than 500, 000 deaths per year [1]. OSCC is characterized by invasive growth, frequent regional metastases, high recurrence, and poor prognosis. Tobacco smoking and alcohol consumption are the main etiological factors in OSCC, although other habits such as betel nut and tobacco chewing have also been implicated in Asian populations. Despite the easy access of the oral cavity to examination, up to $60 \%$ of OSCC cases remained undiagnosed until an advanced clinical stage. Moreover, despite great progress in its diagnosis and combined treatments, including surgical resection, chemotherapy, radiation therapy, and targeted biological agents, the 5-year overall survival rate of OSCC remains less than 50\%; though this can rise significantly to $85.4 \%$ in patients diagnosed with early-staged tumors $[2,3]$. The early detection of OSCC is therefore key to improving patient prognosis and survival rates.

Blood biomarkers have played an important role in diagnosing and surveying oral cancer compared with invasive tissue biopsies [4-6]. However, despite the wealth of blood biomarkers for OSCC such as squamous cell carcinoma antigen (SCCA) [7] and carcinoembryonic antigen (CEA), little progress has been made regarding the treatment and early detection of OSCC [8]. Further clinical investigations are therefore needed to demonstrate the clinical importance of validated predictors of survival, tumor recurrence, lymph node metastasis, and therapy resistance.

Long non-coding RNAs (lncRNAs) are a novel class of RNA transcripts longer than 200 nucleotides and with limited protein-coding potential $[9,10]$. Many reports have shown that IncRNAs play important roles in various biological processes and are dysregulated in many diseases, particularly cancer $[11,12]$. Aberrant expression of serum lncRNAs has been reported to serve as a potential diagnostic or prognostic biomarker in many malignancies, including breast, colon, pancreatic, and lung cancers [13-16].

Circulating RNA in the serum has been used for non-invasive diagnostic applications, instead of tissue biopsies $[17,18]$. Increased levels of alkaline serum ribonuclease (RNase) have been detected in cancer patients, especially those with adenocarcinomas and squamous cell carcinomas. However, recent studies showed that circulating RNAs may be protected from RNase degradation by assembly into extracellular vesicles [19], thus making them remarkably stable [20]. Numerous reports have revealed that several circulating lncRNAs could function as sensitive biomarkers for the early diagnosis of various cancers, such as POU3F3 for esophageal squamous cell carcinoma, HOTTIP-005 and RP11-567G11.1 and their plasma fragments for pancreatic cancer, and H19 for gastric cancer [13, 15, 21]; however, little is known about the early detection of OSCC. In this study, we aimed to screen for differentially expressed IncRNAs in OSCC, and to perform IncRNA microarray analysis to assess the clinical values of these lncRNAs and the conventional protein biomarkers SCCA, tumor-specific growth factor (TSGF), programmed death ligand 1 (PD-L1), and CEA.

\section{Materials and Methods}

\section{Patients and control individuals}

Eighty OSCC patients who had not received prior radiotherapy, chemotherapy, or other antitumor treatment, and 70 healthy control individuals were recruited between September 2014 and November 2016. Tumor tissues and matched non-tumor samples (MNT) were randomly selected more than $1.5 \mathrm{~cm}$ away from patients undergoing surgery at the Department of Oral and Maxillofacial Surgery, NanFang Hospital. Clinicopathological data were also collected at the same time. All OSCC samples were examined by two or more independent pathologists. Tumor stage was determined according to the Union of International Control of Cancer classification. Each sample was placed immediately in RNAwait (SR0020; Solarbio, Beijing, China) and then stored at $-80^{\circ} \mathrm{C}$ until use. Blood samples were collected in dry vacuum tubes and isolated by centrifugation at $5000 \times \mathrm{g}$ for $10 \mathrm{~min}$, followed by further centrifugation at $14,000 \times \mathrm{g}$ for $15 \mathrm{~min}$ to obtain 


\section{Cellular Physiology Cell Physiol Biochem 2018;47:26-38

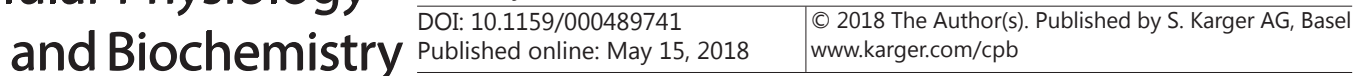

Shao et al.: Circulating Biomarkers in OSCC

serum. All serum samples were stored in RNase-free tubes at $-80^{\circ} \mathrm{C}$ before further analysis. Blood samples with hemolysis were excluded. Sample handling was approved by the hospital ethics committee.

\section{Microarray profiling}

Three paired tissue specimens were obtained from OSCC patients with different clinical stages and differentiated pathologies. All microarray work was performed by KangChen Bio-tech (Shanghai, China) using Arraystar Human LncRNA Microarray V3.0 ( $8 \times 60 \mathrm{~K}$, Rockville, MD, USA), which contains approximately 30,586 IncRNAs and 26, 109 coding transcripts, and is designed for the global profiling of human lncRNAs and protein-coding transcripts. Labeled complementary (c)RNAs were purified using an RNeasy Mini Kit (Qiagen, Germany). The concentration and specific activity of the labeled cRNAs were measured using a NanoDrop ND-1000 spectrophotometer (NanoDrop, USA) and the acquired array images were analyzed using Agilent Feature Extraction software (version 11.0.1.1). Quantile normalization and subsequent data processing were performed using the GeneSpring GX v12.1 software package (Agilent Technologies). After quantile normalization of the raw data, IncRNAs and mRNAs, for which at least three out of six samples had flags in Present or Marginal ("All Targets Value"), were chosen for further data analysis. LncRNAs and mRNAs that were significantly differentially expressed between OSCC patients and controls were identified through fold-change filtering $(\geq 2.0$ or $\leq 0.5)$ and using Linear Models for Microarray Data (LIMMA) (moderated $t$-test, $p<0.05$ ) and multiple hypothesis testing (false discovery rate, FDR $<0.2$ ).

\section{Bioinformatics analysis}

The Gene Ontology (GO) project provides a controlled vocabulary to describe gene and gene-product attributes in any organism (http://www.geneontology.org), covering three domains: Biological Process, Cellular Component, and Molecular Function. Fisher's exact test is used to determine if there is more overlap between the differentially expressed and GO annotation lists than would be expected by chance, with the resulting $p$-value denoting the significance of GO term enrichment among the differentially expressed genes. Pathway analysis involves functional mapping of genes to Kyoto Encyclopedia of Genes and Genomes (KEGG) pathways. The $p$-value (EASE score, Fisher's exact $p$-value or hypergeometric $p$-value) denotes the significance of the pathway correlated with the conditions. The lower the $p$-value for GO or KEGG analysis, the more significant the term or pathway $(p \leq 0.05)$.

\section{Cell culture}

The human squamous cell lines SCC9, SCC15, and SCC25, and the normal oral epithelial cell line HOK were obtained from the Institute of Antibody Engineering, Southern Medical University (Guangzhou, China). Cell lines were maintained in DMEM/F12 medium (Invitrogen, Carlsbad, CA, USA) supplemented with $10 \%$ fetal bovine serum (Life Technologies, Carlsbad, CA, USA). All cells were cultured in a humidified 5\% $\mathrm{CO}_{2}$ incubator at $37^{\circ} \mathrm{C}$. After incubation for 3 days, the cells and media were collected separately for RNA isolation. The conditioned media were processed as described for serum collection.

\section{SCCA, TSGF, PD-L1, and CEA assays}

Serum concentrations of SCCA, TSGF, and PD-L1 were assessed by conventional enzyme-linked immunosorbent assays (ELISAs), according to the manufacturer's protocols. ELISA kits for SCCA (lot: CSB-E09381h), TSGF (lot: CSB-E08843h), and PD-L1 (lot: CSB-E13644h) were purchased from CUSABIO (Wuhan, China). Each test included a standard control (coefficient of variation, CV $<5 \%$ ). Serum CEA level was detected by radioimmunoassay at the Department of Pathology at NanFang Hospital (Guangzhou, China). A cut-off value of $5 \mathrm{ng} / \mathrm{mL}$ was used for CEA. The index tests and reference standards were blinded during detection. No adverse events resulted from performing the index tests. All results here are expressed as the mean \pm standard deviation (SD) of three independent experiments.

\section{Real-time quantitative PCR ( $q P C R$ ) analysis}

RNA was extracted from tissues and cultured cells using TRIzol reagent (Invitrogen), and total RNA was extracted from serum or cell culture media using TRIzol LS Reagent (Ambion, Waltham, MA, USA) following the manufacturer's protocol. Serum was separated from venous blood within $12 \mathrm{~h}$. Total RNA was reverse transcribed into cDNA using a PrimeScript ${ }^{\text {TM }}$ RT reagent kit with gDNA Eraser (RR047A; Takara, Shiga, Japan) according to the manufacturer's instructions. Standard qPCR was carried out using SYBR® 


\section{Cellular Physiology Cell Physiol Biochem 2018:47:26-38

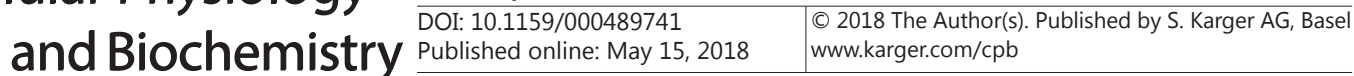 \\ Shao et al.: Circulating Biomarkers in OSCC}

Premix Ex Taq ${ }^{\text {TM }}$ II (RR820A; Takara) in $20 \mu \mathrm{L}$ reactions. Quantitative detection of lncRNA was performed using an ABI Step One Real-Time PCR System (Applied Biosystems, San Diego, CA, USA). Briefly, the mixture was incubated at $95^{\circ} \mathrm{C}$ for $30 \mathrm{~s}$, followed by 40 cycles of $95^{\circ} \mathrm{C}$ for $5 \mathrm{~s}$, and $60^{\circ} \mathrm{C}$ for $30 \mathrm{~s}$. Each sample was analyzed in triplicate and the specificity of each PCR reaction was confirmed by melting-curve analysis. The expression levels of IncRNAs were normalized to the internal control GAPDH, and then calculated using the $2^{-\Delta \Delta C T}$ method.

\section{Statistical analysis}

Data are presented as the mean \pm standard error (SE). Measured data were compared between groups using Student's paired t-tests or analysis of variance. Relationships between two groups were tested by Pearson's or Spearman's correlation analysis. The prediction value was calculated by receiver operating characteristic (ROC) curve and decision curve analyses (DCA). Survival was analyzed using a Cox proportional hazards model. All statistical analyses were performed using SPSS software (version 22; IBM, Armonk, NY, USA) and presented graphically in GraphPad Prism 5.0 (GraphPad, La Jolla, CA, USA). All tests were two-tailed and $p<0.05$ was considered statistically significant.

\section{Results}

\section{Differentially expressed IncRNAs and bioinformatics analysis}

We conducted IncRNA and mRNA expression profiling of three pairs of OSCC and MNT tissues via microarray analysis. In total, 691 lncRNAs (433 upregulated and 258 downregulated) and 816 mRNAs (342 upregulated and 474 downregulated) were found to be differentially expressed (fold change $\geq 2$ in both groups, normalized intensity of each group $\geq 5$ ). Data for the IncRNA microarray were collected from databases including the National Center for Biotechnology Information RefSeq, Ensembl database, University of California, Santa Cruz (UCSC), IncRNA database, Gencode, Ultra Conserved Regions, and other related sources in the published literature. Hierarchical clustering analysis (Fig. 1), scatter and volcano plots were used to visualize differentially expressed lncRNAs (Fig. 2). Based on the location relationship of nearby coding genes, differentially expressed lncRNAs were classified into several types, including 86 natural antisense, 76 intronic antisense, 411 intergenic, 39 exon sense-overlapping,

bidirectional, and 27 intron sense-overlapping.

GO analysis indicated that these gene products were mainly found in the nuclear and extracellular regions (Fig. 3A) and were involved in the biological processes of cell cycle progression, regulating peptidase activity, DNA metabolism, and collagen fibril organization (Fig. 3B). The molecular functions of these genes included ion binding, protein binding, cation binding, and molecular function regulation (Fig. 3C).

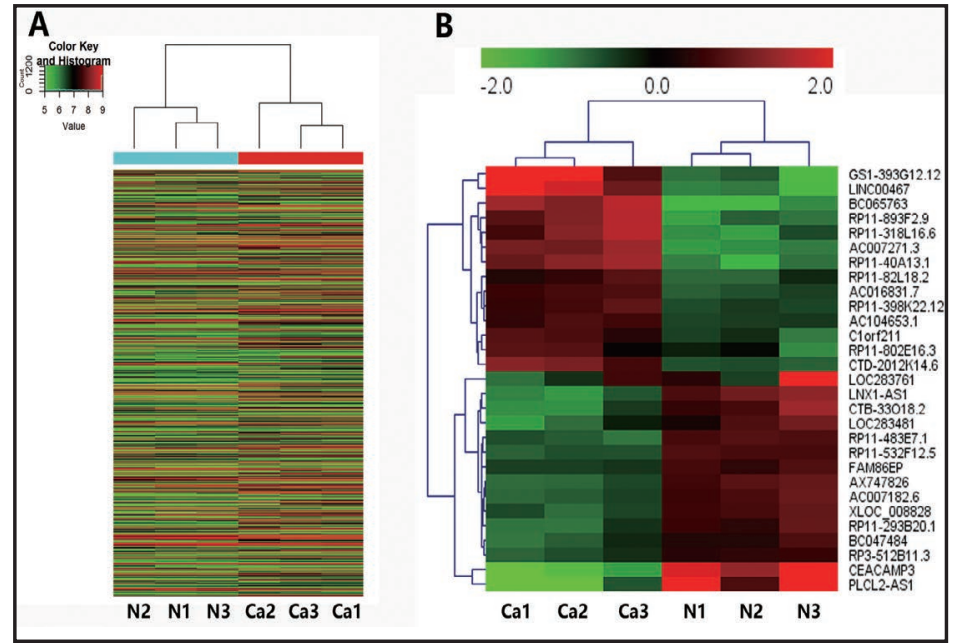

Fig. 1. Microarray profiles. (A) Hierarchical clustering of lncRNA expression profiles in OSCC tissues and MNT. (B) Twenty-nine IncRNAs were significantly dysregulated, with distinct differences between OSCC and adjacent non-tumorous tissues. "Ca", tumor group; "N", normal group. Expression values are represented in shades of red and green, indicating expression above and below the median value across all samples. 
Fig. 2. Microarray profiles. Scatter plot (A) and volcano plot (B) showing the distributions of lncRNAs. After normalization, the distributions of the log2 ratios were nearly the same. X- and y-axes in the scatter plot show the averaged normalized signal values of the group ( $\log 2$-scaled). Green lines in the scatter plot and volcano plot represent the default significant foldchange (2.0).

Pathway analysis based on the KEGG database identified a total of 26 pathways with significant differences in gene expression between the OSCC and MNT tissues. The pathway terms for the 10 highest enrichment scores are shown in Fig. 3D. The ErbB (hsa04012), adherens junctions (hsa04520), and TNF pathways (hsa04668) are associated with carcinogenesis.

QPCR validation of significantlydysregulated IncRNAs in OSCC tissues and $M N T$

Among the aberrantly expressed IncRNAs, 29 were significantly dysregulated (absolute fold change $>2.0$, moderated $t$-test $p<0.05$, $\mathrm{FDR}<0.2$, and at least one out of two groups with flags in Present or Marginal) (Table 1). We verified the microarray data by validating the gene expression levels by qPCR in 30 pairs of OSCC tissues and MNT. The expression patterns of 25 lncRNAs were consistent with the microarray data (Fig. 4), demonstrating their reliability, while the other four lncRNAs were excluded because their expression patterns were inconsistent with the microarray data. AC007271.3, AC007182.6, LOC283481, and RP11-893F2.9 were significantly dysregulated in OSCC tissues compared with MNT (Fig. 5A).

Predictive potential of AC007271.3 for OSCC

To test the stability of the four significantly dysregulated IncRNAs in OSCC serum, we subjected serum samples to harsh conditions including longer incubation at room tempera- 
Fig. 3. GO and pathway analyses to explore potential functions of the IncRNAs dysregulated in OSCC tissues and MNT. (A) GO analysis showed that the gene products were mainly located in the nuclear and extracellular regions. The top 10 participating biological processes (B) and the molecular functions (C) of targeted genes are listed. (D) Pathway analysis indicated that the genes were involved in pathways of Chagas disease, sphingolipid signaling, systemic lupus erythematosus, adherens junc-

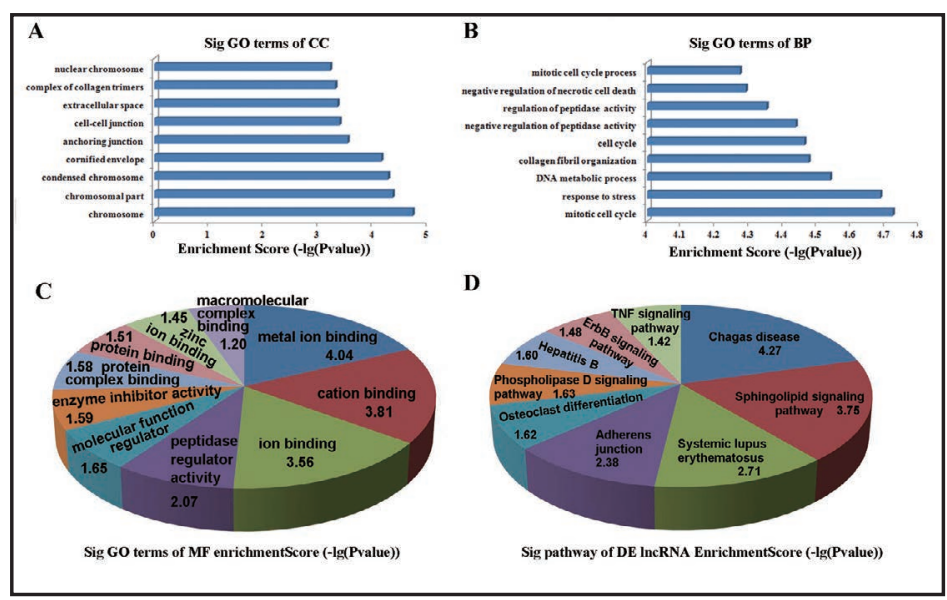
tions, osteoclast differentiation, phospholipase D signaling, hepatitis B, ErbB signaling, and TNF signaling.

ture, repeated freeze-thaw cycles, and RNase A digestion. These treatments had little or no impact on the serum concentrations of AC007271.3 and LOC283481, which were therefore selected for further study. In contrast, AC007182.6 and RP11893F2.9 were degraded by these treatments and were not studied further (Fig. 5C).

We determined AC007271.3 and LOC283481 expression in three OSCC cell lines (SCC9, SCC15, and SCC25), one human oral epithelial cell line (HOK), and in conditioned culture media. Compared with HOK cells, AC007271.3 was up-regulated approximately 2-fold in OSCC cell lines, and the AC007271.3

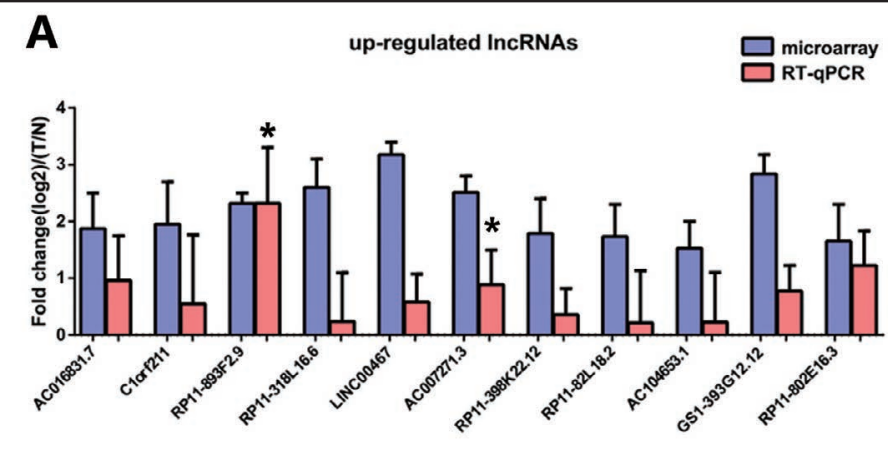

B down-regulated IncRNAs

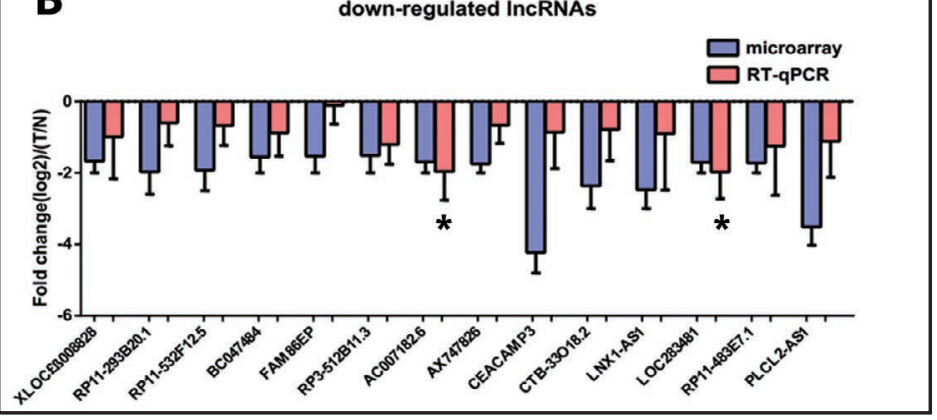

Fig. 4. Validation of significantly dysregulated lncRNAs by qPCR. Expression patterns for 25 lncRNAs are shown, including 11 upregulated (A) and 14 downregulated (B), which were consistent with microarray data. level in the culture medium from OSCC cell lines increased with increasing cell count $\left(0.5-2 \times 10^{6}\right.$ cells per well) and incubation time (1-3 days) (Fig. 5E). These results suggested that serum AC007271.3 may be secreted by tumor cells. In contrast, LOC283481 expression levels did not differ significantly between OSCC and HOK cells, and this lncRNA was therefore excluded from further studies.

Serum AC007271.3 expression differed significantly between before and 14 days after surgery ( $p=0.002$ ) (Fig. 5D), and there was a significant correlation between serum and tissue AC007271.3 levels $(r=0.639, p<0.001)$. We therefore inferred that serum AC007271.3

\section{KARGER}




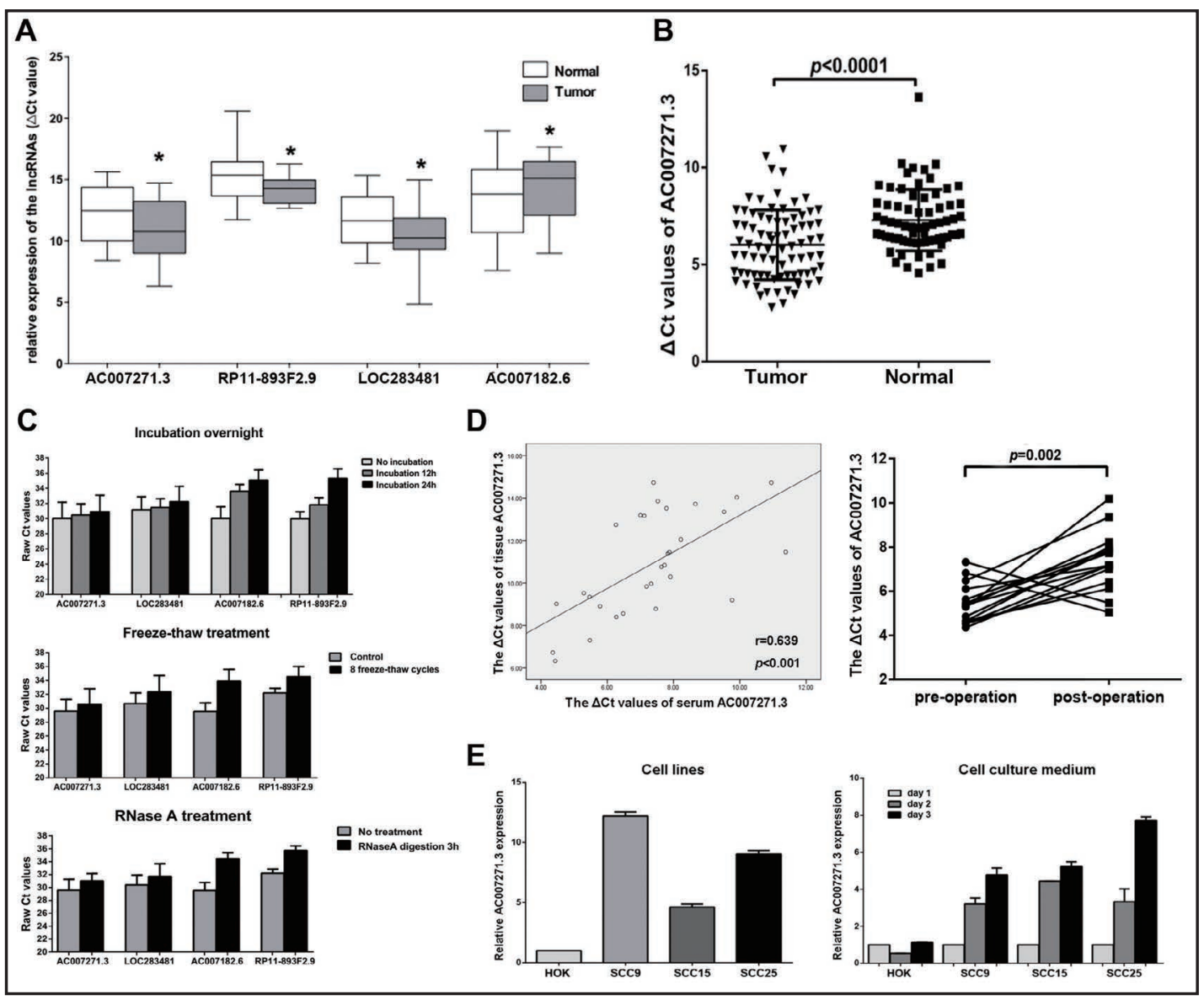

Fig. 5. Validation of OSCC-related lncRNA expression as a circulating biomarker. (A) Differential expression of IncRNAs in OSCC tissues compared with MNT. (B) Serum AC007271.3 expression was measured in 80 clinical samples and 70 cancer-free controls. Data are shown as $\Delta$ Ct values normalized to GAPDH, with smaller $\Delta$ Ct values indicating higher expression. (C) Serum AC007271.3 and LOC283481 levels remained stable, even under harsh conditions ( $\mathrm{p}<0.05)$. (D) Spearman's rank correlation scatter plot of AC007271.3 levels in OSCC tissues and serum. Wilcoxon's test was used to compare paired serum samples before and 14 days after surgery. Data are presented as $\Delta$ Ct values normalized to GAPDH. (E) Expression of AC007271.3 in OSCC and HOK cells ( $\mathrm{p}<0.05)$. AC007271.3 levels in OSCC cell culture medium incubated for 0,1 , and 2 days $(\mathrm{p}<0.05$, compared with HOK). Data presented as relative lncRNA fold changes.

levels were coincident with its tumor expression and that serum AC007271.3 was secreted by OSCC cells.

Serum AC007271.3 expression was also significantly upregulated in 80 OSCC patients compared with 70 healthy controls $(p<0.0001$, Fig. $5 \mathrm{~B})$, indicating that it is was a potential OSCC biomarker.

Correlations between circulating AC007271.3, SCCA, TSGF, CEA, and PD-L1 levels and clinical characteristics

We explored the correlations between clinical characteristics and serum AC007271.3, SCCA, TSGF, CEA, and PD-L1 levels in OSCC patients following qPCR analysis of serum samples from 80 OSCC patients. AC007271.3 overexpression was significantly associated with degree of pathological differentiation $(p=0.022)$, clinical stage $(p=0.005)$, lymphatic metastasis $(p<0.002)$, and smoking history $(p=0.011)$ but not with age, sex, or alcohol history (Table 2). Serum SCCA, TSGF, and PD-L1 levels also differed significantly between OSCC cases at different tumor stages, differentiation stages, and with or without lymphatic metastasis.

\section{KARGER}


However, there was no significant association between CEA and any clinicopathological parameters.

\section{Combined detection of serum OSCC tumor markers}

To determine if AC007271.3, SCCA, TSGF, CEA, and/or PD-L1 were potential tumor markers for OSCC, we measured their serum levels in 80 OSCC patients and 70 healthy controls by qPCR and analyzed the ROC curves and area under the ROC curves (AUC), as well as the correlations among the five potential tumor markers (Fig. 6A).

The ROC curves illustrated a strong separation between OSCC patients and controls for AC007271.3 (AUC, 0.873; 95\% confidence interval (CI), 0.815-0.931; $p<0.001$ ), compared with the classic tumor markers SCCA (AUC, $0.719 ; 95 \%$ CI, $0.635-0.804 ; p<0.001$ ) and TSGF (AUC, 0.648; 95\%CI, 0.554-0.742; $p<0.05$ ). Moreover, serum AC007271.3, SCCA, and TSGF levels could discriminate between OSCC and normal controls with sensitivities of $77.6 \%$, $55.0 \%$, and $63.3 \%$ and specificities of $84.5 \%, 93.3 \%$, and $66.7 \%$, respectively, while serum PD-L1 levels were less specific $(39.1 \%)$ and CEA levels were less sensitive $(40.0 \%)$ for the detection of OSCC. We further improved the diagnostic accuracy by using parallel combinations of these biomarkers; i.e., samples were defined as positive for OSCC if any markers in the combination were above the cut-off value.

Interestingly, all three markers (AC007271.3, SCCA, and TSGF) in the combined indices exceeded 1 or 2 with regard to OSCC detection (AUC, 0.917;

Table 2. Correlations between clinical characteristics and serum AC007271.3 and traditional biomarkers in OSCC patients. Results are mean \pm SD. ${ }^{\mathrm{a}} \mathrm{p}<0.05,{ }^{\mathrm{b}} \mathrm{p}<0.01,{ }^{\mathrm{c}} \mathrm{p}<0.001$ vs control group

\begin{tabular}{|c|c|c|c|c|c|c|}
\hline Characteristics & $\mathrm{n}$ & $\begin{array}{c}\text { AC007271.3 } \\
(\Delta \text { Ct value })\end{array}$ & $\begin{array}{c}\text { SCCA } \\
\text { (ng/ml) }\end{array}$ & $\begin{array}{c}\text { TSGF } \\
(\mathrm{U} / \mathrm{ml})\end{array}$ & $\begin{array}{c}\text { CEA } \\
(\mathrm{ng} / \mathrm{ml})\end{array}$ & $\begin{array}{c}\text { PD-L1 } \\
\text { (pg/ml) }\end{array}$ \\
\hline \multicolumn{7}{|l|}{ Age (years) } \\
\hline$<60$ & 50 & $5.848 \pm 0.239$ & $1.27 \pm 1.28$ & $54.36 \pm 7.83$ & $1.79 \pm 0.62$ & $18.49 \pm 4.75$ \\
\hline$\geq 60$ & 30 & $6.127 \pm 0.365$ & $0.90 \pm 0.55$ & $57.23 \pm 8.79$ & $1.73 \pm 0.73$ & $21.57 \pm 4.11$ \\
\hline \multicolumn{7}{|l|}{ Gender } \\
\hline Male & 46 & $5.907 \pm 0.285$ & $1.13 \pm 1.23$ & $56.81 \pm 8.64$ & $1.67 \pm 0.66$ & $19.18 \pm 4.79$ \\
\hline Female & 34 & $6.015 \pm 0.281$ & $1.09 \pm 0.73$ & $53.31 \pm 7.29$ & $1.93 \pm 0.63$ & $20.43 \pm 4.62$ \\
\hline \multicolumn{7}{|l|}{ Smoking statues } \\
\hline Yes & 36 & $5.421 \pm 0.289^{a}$ & $1.12 \pm 1.34$ & $54.94 \pm 7.73$ & $1.73 \pm 0.70$ & $19.11 \pm 4.91$ \\
\hline No & 44 & $6.388 \pm 0.265$ & $1.10 \pm 0.72$ & $56.17 \pm 9.05$ & $1.81 \pm 0.60$ & $20.38 \pm 4.47$ \\
\hline \multicolumn{7}{|c|}{ Alcohol consumption } \\
\hline Yes & 45 & $5.830 \pm 0.294$ & $1.31 \pm 1.27$ & $55.01 \pm 8.13$ & $1.73 \pm 0.73$ & $19.11 \pm 4.91$ \\
\hline No & 35 & $6.111 \pm 0.267$ & $0.85 \pm 0.55$ & $56.04 \pm 8.55$ & $1.82 \pm 0.56$ & $20.38 \pm 4.47$ \\
\hline \multicolumn{7}{|c|}{ TNM classification } \\
\hline I and II & 47 & $6.417 \pm 0.271^{\mathrm{a}}$ & $1.11 \pm 1.21^{\mathrm{a}}$ & $53.49 \pm 7.58$ & $1.72 \pm 0.72$ & $19.13 \pm 4.86$ \\
\hline III and IV & 33 & $5.292 \pm 0.264^{b}$ & $1.11 \pm 0.75^{\mathrm{b}}$ & $58.29 \pm 8.54^{a}$ & $1.83 \pm 0.56$ & $20.44 \pm 4.52^{\mathrm{a}}$ \\
\hline \multicolumn{7}{|c|}{ Lymphatic metastasis } \\
\hline $\mathrm{N}_{0}$ & 58 & $6.337 \pm 0.240^{b}$ & $1.30 \pm 1.24 \mathrm{a}$ & $53.16 \pm 7.36$ & $1.70 \pm 0.72$ & $19.31 \pm 4.96$ \\
\hline $\mathrm{N}_{1}-\mathrm{N}_{3}$ & 22 & $4.941 \pm 0.276^{c}$ & $0.81 \pm 0.49 b$ & $59.14 \pm 8.44^{\mathrm{a}}$ & $1.88 \pm 0.54$ & $20.23 \pm 4.37 \mathrm{a}$ \\
\hline \multicolumn{7}{|l|}{ Differentiation } \\
\hline Well/moderate & 49 & $6.319 \pm 0.223 \mathrm{a}$ & $0.84 \pm 0.53^{a}$ & $53.26 \pm 8.43$ & $1.71 \pm 0.76$ & $19.03 \pm 4.83$ \\
\hline poor & 31 & $5.375 \pm 0.353^{b}$ & $1.55 \pm 1.46^{b}$ & $58.47 \pm 7.14^{a}$ & $1.80 \pm 0.59$ & $21.38 \pm 4.11^{a}$ \\
\hline
\end{tabular}

Fig. 6. ROC curve analysis of the predictive power of serum biomarkers in OSCC. (A) The area under the ROC curve was estimated in 80 OSCC patients and 70 controls. ROC curves for the diagnostic strength of AC007271.3, SCCA, TSGF, CEA, or PD-L1 for identifying OSCC (AUC: CEA, 0.588; TSGF, 0.648; SCCA, 0.719; PD-L1, 0.641). (B) Diagnostic outcomes for serum AC007271.3, SCCA, TSGF, CEA, or PD-L1 combinations in OSCC diagnoses. (C, D) ROC curve analyses for the discriminative ability between early-stage OSCC cases and controls.
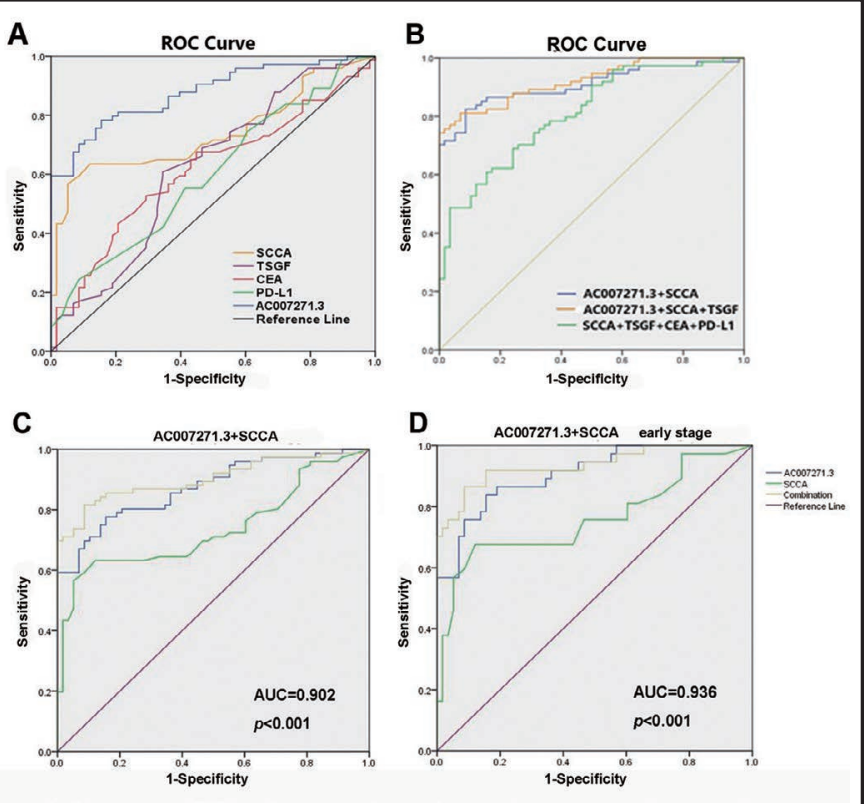
95\%CI, 0.872-0.962; $p<0.001)$. This suggested that the optimal cut-off values determined by ROC curve analysis could improve OSCC diagnostics when used together, thus promoting clinical screening and OSCC diagnosis (Table 3, Fig. 6B). DCA was used to evaluate the reliability of the predictions using these clinical biomarkers alone or in combination, taking into account adjustments for polymorphisms, age at diagnosis, gender, smoking status, and alcohol consumption, and showed a moderate benefit of incorporating AC007271.3, SCCA, and TSGF with clinical variables for high threshold probability (Fig. 7).

Moreover, the combination of AC007271.3 and SCCA could be used to distinguish between early-stage OSCC patients and controls to a clinically satisfactory degree compared with conventional tumor markers (AUC, 0.936; 95\% $\mathrm{CI}$, 0.882-0.990; $p<0.001$ ) (Fig. 6C, D).

Postoperative tumor recurrence and metastasis are major causes of death in OSCC patients. Twelve patients in the current study died (two of the twelve who died experienced recurrence) during the follow-up period (6-37 months). The survival time for each patient was defined as the interval between the date of definitive resection and the date of last followup or death. Multivariate survival analysis using the Cox proportional hazards model showed that the combination of serum AC007271.3 and SCCA was an independent prognostic factor for survival in patients with OSCC (Table 4). Survival curves are shown
Table 3. Evaluation of diagnostic values of different marker combinations in 80 OSCC cases. ROC curves were created to evaluate the power of the biomarkers to predict OSCC. Abbreviations: YI, Youden's index; $\mathrm{Cl}$, confidence interval; Sensitivity, true positive/patients $\times 100 \%$; Specificity, true negative/normal $\times 100 \%$

\begin{tabular}{lcccc}
\hline Diagnostic value & AUC $(95 \% \mathrm{CI})$ & Sensitivity & Specificity & YI \\
\hline AC007271.3 & $0.873(0.815-0.931)$ & $77.5 \%$ & $84.5 \%$ & $62.0 \%$ \\
SCCA & $0.719(0.635-0.804)$ & $55.0 \%$ & $93.3 \%$ & $48.3 \%$ \\
TSGF & $0.648(0.554-0.742)$ & $63.3 \%$ & $66.7 \%$ & $30.0 \%$ \\
PD-L1 & $0.641(0.499-0.783)$ & $84.1 \%$ & $39.1 \%$ & $23.2 \%$ \\
CEA & $0.588(0.494-0.683)$ & $40.0 \%$ & $78.3 \%$ & $18.3 \%$ \\
AC007271.3+SCCA & $0.902(0.849-0.955)$ & $82.5 \%$ & $91.4 \%$ & $73.9 \%$ \\
AC007271.3+TSGF & $0.888(0.835-0.940)$ & $65.3 \%$ & $98.3 \%$ & $63.6 \%$ \\
AC007271.3+SCCA+TSGF & $0.917(0.872-0.962)$ & $80.0 \%$ & $93.1 \%$ & $73.1 \%$ \\
SCCA+TSGF+PD-L1+CEA & $0.809(0.739-0.879)$ & $48.7 \%$ & $96.7 \%$ & $45.4 \%$ \\
\hline
\end{tabular}

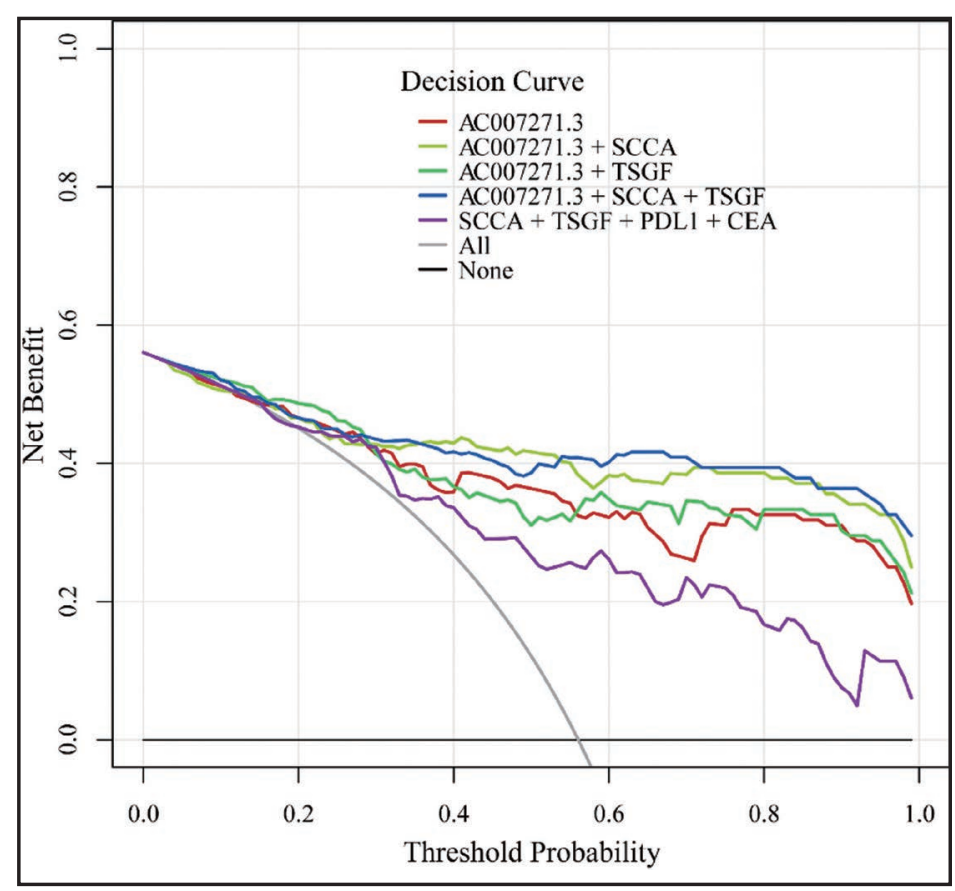

Fig. 7. Decision curve analysis for the predictive power of serum biomarkers AC007271.3, SCCA, TSGF, CEA and PD-L1 risk-prediction models in OSCC.

Table 4. Multivariate analysis using a Cox regression model for all OSCC patients. Abbreviations: aHR, adjusted hazard ratio; $\mathrm{Cl}$, confidence interval. Adjusted for age, gender, smoking status, and alcohol consumption

\begin{tabular}{lcccc}
\hline Prognostic factor & Wald $\chi^{2}$ & P value & aHR & $95 \%$ CI \\
\hline AC007271.3 & 3.503 & 0.061 & 3.083 & $0.9482-10.02$ \\
SCCA & 0.052 & 0.819 & 1.144 & $0.3718-3.7593$ \\
TSGF & 0.760 & 0.383 & 1.856 & $0.4620-7.459$ \\
PD-L1 & 1.414 & 0.234 & 0.471 & $0.1360-1.630$ \\
AC007271.3+SCCA & 5.385 & 0.0203 & 4.773 & $1.301-12.51$ \\
LN metastasis & 5.064 & 0.0244 & 4.919 & $1.228-19.70$ \\
\hline
\end{tabular}


in Fig. 8 to show the overall survival of the 80 OSCC patients.

\section{Discussion}

Increasing evidence has shown that IncRNAs play important roles in histone acetylation, gene methylation, posttranscriptional translation, and other biological processes and have

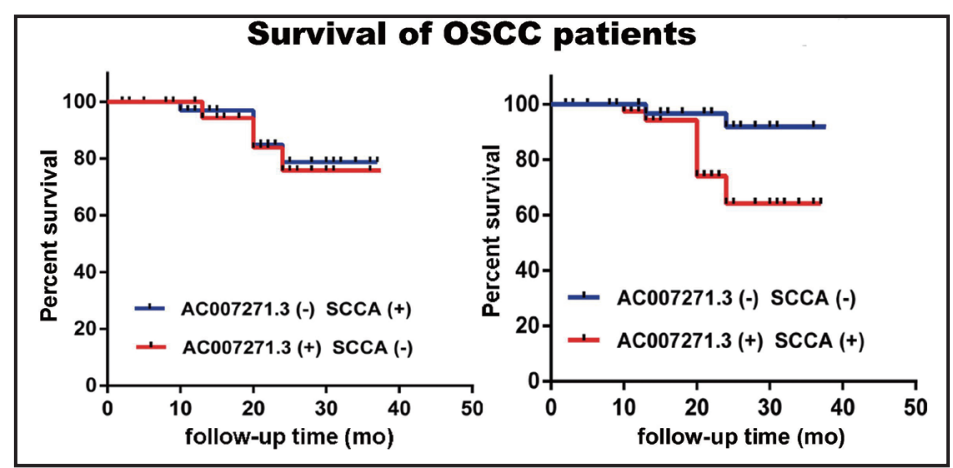

Fig. 8. Analysis of overall survival curves for 80 OSCC patients. KaplanMeier survival curves in OSCC patients using a Cox proportional hazards model for multivariate regression analysis. emerged as key regulators of tumor development and progression. Several studies to date have implicated various IncRNAs in the progression of OSCC, including UCA1, NKILA, MALAT1, HOTAIR, FTH1P3, and TUC338 [21-23]. However, despite this progress, the precise functions and mechanisms of IncRNAs in OSCC remain unclear. We therefore compared the gene expression profiles of OSCC and paired MNT tissues using microarray analysis, to identify lncRNAs with functions in OSCC carcinogenesis.

The microarray results suggested that the OSCC characteristics of invasive growth, frequent regional metastases, high recurrence, and poor prognosis could be related to differential expression of IncRNAs. GO analysis of the dysregulated lncRNAs indicated that their gene products were typically found in the nuclear and extracellular regions and that the enriched lncRNAs mainly regulated biological processes such as cell cycle progression, peptidase activity, DNA metabolism, and collagen fibril organization. The target gene pool mostly included ion binding, protein binding, cation binding, and molecular function regulators, indicating that the dysregulated lncRNAs may play vital roles in cell adhesion and migration in OSCC. Furthermore, pathway analysis showed that the dysregulated IncRNAs participated in 26 signaling pathways, of which several were associated with OSCC carcinogenesis, including ErbB signaling (hsa04012), adherens junctions (hsa04520), and TNF signaling (hsa04668) pathways.

Recent studies have shown that IncRNAs can be released from tumor tissues into the circulation and are detectable in the blood, urine, and sputum of cancer patients. This suggests that some cell-free lncRNAs carry regulatory signals involved in pathological conditions such as tumor occurrence, invasion, and metastasis, and might thus be potential biomarkers for cancer [24-26]. Indeed, researchers have identified three circulating lncRNAs, Linc00152, CFLAR-AS1, and POU3F3, as potential biomarkers for predicting the development of human esophageal squamous cell carcinoma [27]. Furthermore, levels of the circulating lncRNAs AK001058, INHBA-AS1, MIR4435-2HG, UCA1, and CEBPA-AS1 were significantly higher in gastric cancer patients compared with healthy controls [28]. However, to the best of our knowledge, no clinical studies have reported on circulating lncRNAs in OSCC to date.

We conducted a genome-wide lncRNA screen of OSCC and MNT tissues. All lncRNAs of interest were subjected to qPCR validation, and four (AC007271.3, AC007182.6, LOC283481, and RP11-893F2.9) were identified as significantly dysregulated in tumor compared with MNT tissues. AC007271.3 and LOC283481 remained stable when subjected to harsh conditions, making them better candidate biomarkers for clinical settings. Furthermore, serum AC007271.3 levels appeared to reflect tumor expression levels, providing strong evidence to suggest that OSCC-related IncRNAs could be released into the circulation. Overall, these results suggest that IncRNA expression profiles could be used as diagnostic markers for OSCC. Interestingly, most OSCC patients had significantly decreased AC007271.3 levels after surgery ( $p=0.002)$. We further investigated the correlations between AC007271.3 expression 


\section{Cellular Physiology Cell Physiol Biochem 2018;47:26-38

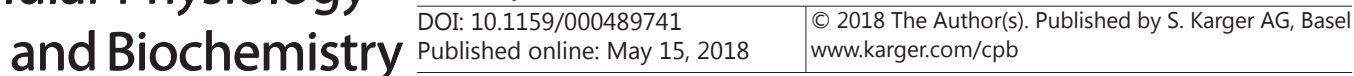 \\ Shao et al.: Circulating Biomarkers in OSCC}

and clinicopathological characteristics, and we showed that high AC007271.3 levels were correlated with smoking history, pathological differentiation, lymphatic metastasis, and advanced tumor stage, which corresponded well with our in vitro experiments. Other recent work similarly reported that secreted IncRNAs were associated with tumor stage in the detection of gastric cancer [29].

New methods for the early detection of OSCC are urgently needed to improve patient outcomes; however, no effective early diagnostic biomarkers have yet been identified. Serum biomarkers such as SCCA, TSGF, CEA, and PD-1 have been used as clinical biomarkers for the early diagnosis of many tumors, because they can be detected with high levels of sensitivity and specificity. However, although recent studies showed that serum SCCA was the best available biomarker with a sensitivity of $80 \%$ in OSCC patients, its specificity was less than $60 \%$ [30-32]. We therefore tested the diagnostic abilities of combinations of these biomarkers for OSCC and showed that the combination of AC007271.3, SCCA, and TSGF discriminated between OSCC and controls with high sensitivity (80.0\%) and specificity (93.1\%). Optimal cut-off values based on ROC curve analysis showed that this combination could facilitate the clinical screening and diagnosis of OSCC. Moreover, DCA of prediction reliability revealed a moderate benefit to incorporating clinical variables to achieve a high threshold probability in the presence of polymorphisms. These analyses indicate the need to detect AC007271.3, SCCA, and TSGF in patients with suspected OSCC. However, TSGF is also a wide-spectrum biomarker with increased serum levels in acute inflammation, collagen diseases, and other clinical conditions. Our ROC and survival analyses thus showed that the combination of AC007271.3 and SCCA was most suitable for the early diagnosis of OSCC.

Levels of traditional cancer biomarkers are generally affected by many other diseases and factors. In this preliminary work, we showed that serum lncRNA levels are associated with the pathogenesis of OSCC and that the combined detection of lncRNAs with traditional biomarkers (SCCA and TSGF) is important for the determination and early diagnosis of OSCC. However, further large-scale investigations are required to confirm these findings and to explore the biological functions and molecular mechanisms of AC007271.3 in the pathogenesis of OSCC.

\section{Acknowledgements}

This study was supported by the Science and Technology Planning Project of Guangdong Province (Grant Number: 2017A020215181 and Grant Number: 2014A020212440), and the National Natural Science Foundation of China (Grant Number: 81472536). Microarray experiments were performed by KangChen Bio-tech (Shanghai, China). We thank Liwen Bianji, Edanz Editing China (www.liwenbianji.cn/ac), for editing the English text of a draft of this manuscript.

\section{Disclosure Statement}

The authors declare that they have no conflict of interests in relation to the contents of this article.

\section{References}

-1 Matsukawa S, Morita K, Negishi A, Harada H, Nakajima Y, Shimamoto H, Tomioka H, Tanaka K, Ono M, Yamada T, Omura K: Galectin-7 as a potential predictive marker of chemo-and/or radio-therapy resistance in oral squamous cell carcinoma. Cancer Med 2014;3:349-361.

-2 Yamamoto N, Sato K, Yamauchi T, Suzuki T, Osaka R, Kin M, Yoshida Y, Noguchi S, Ishizaki K, Takano M, Katakura A, Tanaka Y, Shibahara T, Takano N: A 5-year activity report from the Oral Cancer Center, Tokyo Dental College. Bull Tokyo Dent Coll 2013;54:265-273. 


\section{Cellular Physiology Cell Physiol Biochem 2018;47:26-38 \begin{tabular}{l|l} 
DOI: 10.1159/000489741 & and Biochemistry \\
Publisnec onnne: 1 Vay 15, 2018 & $\begin{array}{l}\text { O 2018 The Author(s). Published by S. Karger AG, Basel } \\
\text { www.karger.com/cpb }\end{array}$
\end{tabular}}

Shao et al.: Circulating Biomarkers in OSCC

3 Takahashi H, Yanamoto S, Yamada S, Umeda M, Shigeta T, Minamikawa T, Shibuya Y, Komori T, Shiraishi T, Asahina I, Yokoo S, Ri S: Effects of postoperative chemotherapy and radiotherapy on patients with squamous cell carcinoma of the oral cavity and multiple regional lymph node metastases. Int. J. Oral Maxillofac Surg 2014;43:680-685.

4 Khurshid Z, Zohaib S, Najeeb S, Zafar MS, Slowey PD, Almas K: Human Saliva Collection Devices for Proteomics: An Update. Int J Mol Sci 2016;17: E846.

-5 Jou YJ, Hua CH, Lin CD, Lai CH, Huang SH, Tsai MH, Kao JY, Lin CW: S100A8 as potential salivary biomarker of oral squamous cell carcinoma using nanoLC-MS/MS. Clin Chim Acta. 2014;436:121-129.

-6 Bigler LR, Streckfus CF, Dubinsky WP: Salivary biomarkers for the detection of malignant tumors that are remote from the oral cavity. Clin Lab Med 2009;29:71-85.

7 Czerninski R, Basile JR, Kartin-Gabay T, Laviv A, Barak V: Cytokines and tumor markers in potentially malignant disorders and oral squamous cell carcinoma: apilotstudy. Oral Dis 2014;20:477-481.

-8 da Silva SD, Ferlito A, Takes RP, Brakenhoff RH, Valentin MD, Woolgar JA, Bradford CR, Rodrigo JP, Rinaldo A, Hier MP, Kowalski LP: Advances and applications of oral cancer basic research. Oral Oncology 2011;47:783-791.

-9 Nagano T, Fraser P: No-nonsense functions for long noncoding RNAs. Cell 2011;145:178-181.

10 Ng SY, Lin L, Soh BS, Stanton LW: Long noncoding RNAs in development and disease of the central nervous system. Trends Genet 2013;29:461-468.

-11 Ren C, Liu Q, Wei Q, Cai W, He M, Du Y, Xu D, Wu Y, Yu J: Circulating miRnAs as Potential Biomarkers of AgeRelated Macular Degeneration. Cell Physiol Biochem 2017;41:1413-1423.

12 González-Ramírez I, Soto-Reyes E, Sánchez-Pérez Y, Herrera LA, García-Cuellar C: Histones and long noncoding RNAs: the new insights of epigenetic deregulation involved in oral cancer. Oral Oncol 2014;50:691695.

13 Yan J, Zhang Y, She Q, Li X, Peng L, Wang X, Liu S, Shen X, Zhang W, Dong Y, Lu J, Zhang G: Long Noncoding RNA H19/miR-675 Axis Promotes Gastric Cancer via FADD/Caspase 8/Caspase 3 Signaling Pathway. Cell Physiol Biochem 2017;42:2364-2376.

14 Serratì S, De Summa S, Pilato B, Petriella D, Lacalamita R, Tommasi S, Pinto R: Next-generation sequencing: advances and applications in cancer diagnosis. Onco Targets Ther 2016;9:7355-7365.

15 Zhao L, Sun H, Kong H, Chen Z, Chen B, Zhou M: The Lncrna-TUG1/EZH2 Axis Promotes Pancreatic Cancer Cell Proliferation, Migration and EMT Phenotype Formation Through Sponging Mir-382 Cell Physiol Biochem 2017;42:2145-2158.

16 Wang P, Lu S, Mao H, Bai Y, Ma T, Cheng Z, Zhang H, Jin Q Zhao J, Mao H: Identification of biomarkers for the detection of early stage lung adenocarcinoma by microarray profiling of long noncoding RNAs. Lung Cancer 2015;88:147-153.

17 Pendlebury A, Hannan NJ, Binder N, Beard S, Mcgauran M, Grant P, Tong S, Whitehead CL: The circulating microRNA-200 family in whole blood are potential biomarkers for high-grade serous epithelial ovarian cancer. Biomed Rep 2017;6:319-322.

-18 Zhang K, Wang YW, Wang YY, Song Y, Zhu J, Si PC, Ma R: Identification of microRNA biomarkers in the blood of breast cancer patients based on microRNA profiling. Gene 2017;619:10-20.

-19 Tkach M, Théry C: Communication by Extracellular Vesicles: Where We Are and Where We Need to Go. Cell 2016;164: 1226-1232.

20 Chretien PB, Matthews W Jr, Twomey PL: Serum ribonucleases in cancer: relation to tumor histology. Cancer 1973;31:175-179.

21 Tong YS, Wang XW, Zhou XL, Liu ZH, Yang TX, Shi WH, Xie HW, Lv J, Wu QQ, Cao XF: Identification of the long non-coding RNA POU3F3 in plasma as a novel biomarker for diagnosis of esophageal squamous cell carcinoma. Mol Cancer 2015;14:3.

22 Nohata N, Abba MC, Gutkind JS: Unraveling the oral cancer lncRNAome: Identification of novel lncRNAs associated with malignant progression and HPV infection. Oral Oncol 2016;59:58-66.

23 Pan F, Yao J, Chen Y, Zhou C, Geng P, Mao H, Fang X: A novel long non-coding RNA FOXCUT and mRNA FOXC1 pair promote progression and predict poor prognosis in esophageal squamous cell carcinoma. Int J Clin Exp Pathol 2014;7:2838-2849.

24 Zhang A, Zhao JC, Kim J, Fong KW, Yang YA, Chakravarti D, Mo YY, Yu J: LncRNA HOTAIR Enhances the Androgen-Receptor-Mediated Transcriptional Program and Drives Castration-Resistant Prostate Cancer. Cell Rep 2015;13:209-221. 


\section{Cellular Physiology Cell Physiol Biochem 2018;47:26-38

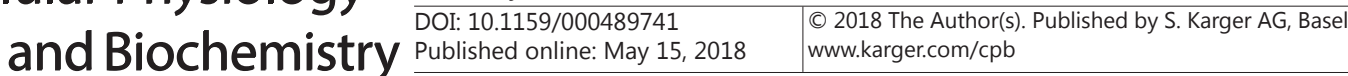 \\ Shao et al.: Circulating Biomarkers in OSCC}

-25 Sun Z, Nie X, Sun S, Dong S, Yuan C, Li Y, Xiao B, Jie D, Liu Y: Long Non-Coding RNA MEG3 Downregulation Triggers Human Pulmonary Artery Smooth Muscle Cell Proliferation and Migration via the p53 Signaling Pathway. Cell Physiol Biochem 2017;42: 2569-2581.

-26 Zhu YP, Bian XJ, Ye DW, Yao XD, Zhang SL, Dai B, Zhang HL, Shen YJ: Long noncoding RNA expression signatures of bladder cancer revealed by microarray. Oncol Lett 2014;7:1197-1202.

27 Hu HB, Jie HY, Zheng XX: Three Circulating LncRNA Predict Early Progress of Esophageal Squamous Cell Carcinoma. Physiol Biochem 2016;40:117-125.

-28 Ke D, Li H, Zhang Y, An Y, Fu H, Fang X, Zheng X: The combination of circulating long noncoding RNAs AK001058, INHBA-AS1, MIR4435-2HG, and CEBPA-AS1 fragments in plasma serve as diagnostic markers for gastric cancer. Oncotarget. 2017;8:21516-21525.

29 Zhang K, Shi H, Xi H, Wu X, Cui J, Gao Y: Genome-Wide IncRNA Microarray Profiling Identifies Novel Circulating lncRNAs for Detection of Gastric Cancer 2017;7:213-227.

30 Radhika T, Jeddy N, Nithya S, Muthumeenakshi RM: Salivary biomarkers in oral squamous cell carcinoma An insight. J Oral Biol Craniofac Res 2016;6:S51-54.

-31 Grimm M, Krimmel M, Hoefert S, Kraut W, Calgéer B, Biegner T, Teriete P, Munz A, Reinert S: Monitoring a 'metabolic shift' after surgical resection of oral squamous cell carcinomas by serum lactate dehydrogenase. J Oral Pathol Med 2016;45:346-355.

32 Grimm M, Kraut W, Hoefert S, Krimmel M, Biegner T, Teriete P, Cetindis M, Polligkeit J, Kluba S, Munz A, Reinert S: Evaluation of a biomarker based blood test for monitoring surgical resection of oral squamous cell carcinomas. Clin Oral Investig 2016;20:329-338. 